\title{
SEPARATE EVALUATION OF NONLINEARITY- DUE Q PENALTIES IN LONG-HAUL VERY DENSE WDM OPTICAL SYSTEMS
}

\author{
Livio Paradiso ${ }^{1}$, Pierpaolo Boffi ${ }^{1}$, Lucia Marazzi ${ }^{1}$, Nicola Dalla Vecchia ${ }^{1}$, Massimo \\ Artiglia $^{2}$ and Mario Martinelli, ${ }^{1,3}$ \\ ${ }^{\text {I}}$ CoreCom, via G. Colombo 8120133 Milan Italy, email: paradiso@corecom.it \\ ${ }^{2}$ Pirelli Cavi S.p.A.,viale Sarca 222 Milan Italy, email: massimo.artiglia@pirelli.com \\ ${ }^{3}$ Politecnico di Milano,piazza Leonardo Da Vinci, email: martinelli@corecom.it
}

\begin{abstract}
Nonlinearity-due Q penalties are experimentally evaluated on a 2000-km, 33$\mathrm{GHz}$ spaced DWDM system, dependently on system length and channel spacing. SPM, FWM and XPM, which is found to be the major constrain, are separately addressed.
\end{abstract}

\section{INTRODUCTION}

In long-haul DWDM systems inter-channel nonlinear effects represent a limit in system capacity increase. While NZDS fibers are designed to prevent Four Wave Mixing (FWM), Cross-Phase Modulation (XPM) still represents the major obstacle in increasing transmission systems capacity [1].

XPM was studied both theoretically $[1,2,3,4]$ and experimentally with pumpprobe $[5,6,7,8]$ and multichannel [9] techniques. Pump-probe scheme allows evaluating separately Self-Phase Modulation (SPM) and XPM impairments. In a WDM system, multichannel measurements account for the global effect of the interfering channels, but it is difficult to quantitatively isolate penalties due to XPM only from those due to other 3rd-order nonlinearities.

In this paper nonlinearity-due $Q$ penalties in a DWDM system are experimentally investigated using a 39 spans optical fiber line $2000-\mathrm{km}$ long. For the first time, to the best of our knowledge, SPM, FWM and XPM penalties are separately addressed in a system with $33-\mathrm{GHz}$ channel spacing. Results are 
described and discussed as a function of optical path length and per-channel input power. XPM-due impairment appears to overweight both SPM and FWM.

\section{EXPERIMENTAL SETUP}

The employed DWDM system is $2000 \mathrm{~km}$ long, with 64 power-equalized, 33$\mathrm{GHz}$ spaced, polarization scrambled channels, in the $1543-1560 \mathrm{~nm}$ window. All channels are $10 \mathrm{Gbit} / \mathrm{NRZ}$-IMDD. The total launch power is $12.8 \mathrm{dBm}$. The system employs $55-\mathrm{km}$ fiber spans with $\mathrm{D}=-2.82 \mathrm{ps} /(\mathrm{nm} \mathrm{km})$ at $1550 \mathrm{~nm}$ and a 55 $\mathrm{km}$ Step index fiber for in-line dispersion compensation every 6 spans. Residual dispersion is fully compensated at the receiver end. This periodical dispersion map allows evaluating penalties at different system lengths. Nonlinear effects are excited at different levels by progressively increasing the per-channel power, by turning off some of the propagating channels (channel count ranges from 64 to 18).

SPM is analyzed first: a probe channel operating at $1551.25 \mathrm{~nm}$ propagates without the 32 neighbouring channels, which are turned off (see the optical spectrum in the inset of Figure 1). In this situation only SPM takes place: we experimentally verified that a given channel is not impaired by other channels farer than $133 \mathrm{GHz}$. By progressively turning off the remaining channels, per-channel power and probe Optical Signal to Noise Ratio (OSNR) is increased and the received $\mathrm{Q}$ factor is measured.

Pump and probe measurements are then performed by introducing a pump channel 100, 66 or $33 \mathrm{GHz}$ away from the probe, thus inducing on it XPM additional penalties. Again, received $Q$ factor is measured, while per-channel power is varied by changing the total channel number. EDFA gain can be considered flat over the band relevant for nonlinear effects, thus minimizing errors in evaluating neighbouring channels power. FWM penalties are not observed owing to the fact that only two channels interact with each other. When all neighbouring channels are turned on, FWM adds to SPM and XPM. Again Q measurements are performed as a function of per-channel power and received OSNR: the channels at the far end of the spectrum are turned on/off, leaving the neighbours on. By combining experimental results, it is possible to separately account for nonlinear effects. FWM penalty is found by subtracting SPM and XPM contributions from the total nonlinearity-due impairments. 


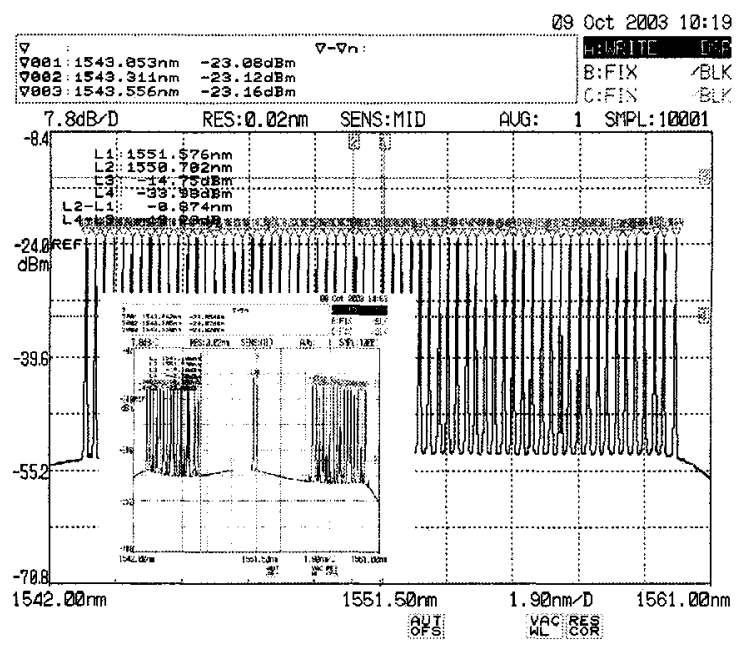

Figure 1. Power-equalized DWDM $64 \mathrm{CH}-33 \mathrm{GHZ}$ spaced spectrum and pump-probe measurement example (in the inset).

\section{MEASUTEMENTS DISCUSSION}

Figure 2 shows the total nonlinearity-due $\mathrm{Q}$ penalties for different system lengths and $33 \mathrm{GHz}$ channel spacing. OSNR is chosen as reference parameter. A $1.5 \mathrm{~dB}$ Q penalty is found for $22.5 \mathrm{~dB}$ OSNR between 1257 and $2000 \mathrm{~km}$ propagation. For higher OSNR (higher per-channel powers) it is possible to compare all the three considered system lengths: total nonlinearity-due $Q$ penalties grow more than linearly with total system length. When residual link dispersion is not compensated, performances worsen and a further $\mathrm{Q}$ penalty is added. Differently from FWM, XPM is influenced both by the dispersion compensation scheme $[4,8]$ and by the residual dispersion value, because residual dispersion allows cumulated nonlinear phase to be further converted into intensity noise. Experiments carried out with a residual dispersion value of $557 \mathrm{ps} /(\mathrm{nmkm})$ lead to an added $Q$ penalty of $1 \mathrm{~dB}$. 


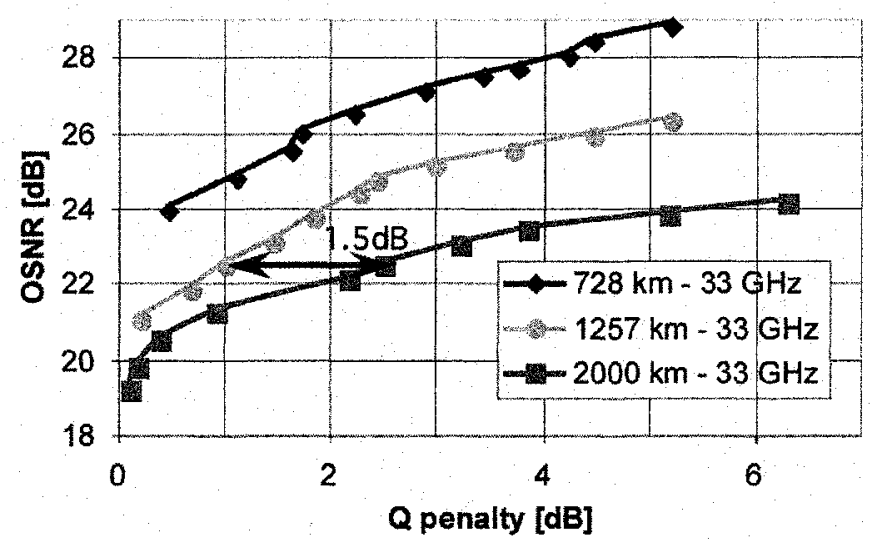

Figure 2. OSNR vs. total nonlinearity-due Q-penalties for different total system length.

The nonlinearity-due $\mathrm{Q}$ penalty is shown in figure 3 as a function of input perchannel power: XPM, FWM and SPM contributions are separately addressed. With $33 \mathrm{GHz}$ channel spacing XPM-due impairment overweighs both SPM and FWM. When channel spacing increases, the amount of XPM, FWM and SPM becomes similar: in particular with $100 \mathrm{GHz}$ channel spacing SPM represents the most important impairment in the $2000 \mathrm{~km}$ system, while XPM always produces a $\mathrm{Q}$ penalty higher than FWM.

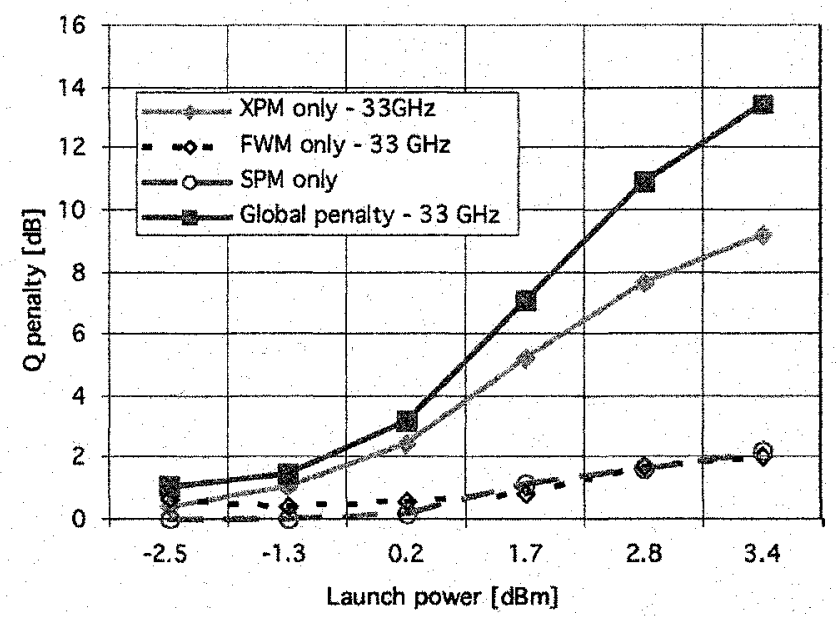

Figure3. Contribution of XPM, FWM and SPM to Q-penalties, for $2000 \mathrm{~km}$ system length.

The system under test is NZDS-fiber based and the dispersion map, typical of submarine systems, is designed to limit FWM penalties. Channels experience a 
significant walk-off before compensation, which is done every 6 spans, thus representing a good trade-off also for XPM penalties reduction [4,8]. At $33 \mathrm{GHz}$ channel spacing, because of the relatively low fiber dispersion $(D=-2.8$ ps/(nmkm)), FWM still produces over $1 \mathrm{~dB}$ Q penalty, as high as SPM, for perchannel launch power over $1.7 \mathrm{dBm}$. XPM proves to be the main nonlinear system impairment when increasing the channel density (see Figure 3).

\section{CONCLUSIONS}

Nonlinearity-due system $Q$ penalties in DWDM multispan systems are experimentally analysed as a function of system length and channel spacing. For the first time to our knowledge $33 \mathrm{GHz}$ spacing is addressed for DWDM systems. SPM, FWM and XPM impairments are separately measured. At $33 \mathrm{GHz}$ spacing XPM proves to be the most severe impairment, whereas at 66 and $100 \mathrm{GHz}$ both XPM and FWM present inter-channel penalties $<1 \mathrm{~dB}$ for relatively high powers, and the most limiting factor in increasing per-channel power and then span length is SPM, as reported in previous works [5].

\section{REFERENCES}

[1] S.Ten, K.M. Ennser, J.M. Grochocinski, S.P. Burtsev, V.L. daSilva, "Comparison of four-wave mixing and cross phase modulation penalties in dense WDM systems", in Proceedings of Optical Fiber Conference 1999, ThC4-1/43

[2] A.V.T. Cartaxo, "Cross-Phase Modulation in Intensity Modulation Direct Detection WDM Systems with Multiple Optical Amplifiers and Dispersion Compensators", J. Light. Tech. 17, 1999, pp. 178-190

[3] S. Betti and M. Giaconi, "Analysis of the Cross-Phase Modulation Effect in WDM Optical Systems", IEEE Phot. Tech. Lett. 13, 2001, pp. 43-45

[4] Bo Xu and Maité Brandt Pearce, "Comparison of FWM and XPM-Induced Crosstalk Using the Volterra Series Transfer Function Method”, J. Light. Tech. 21, 2003, pp. 4053

[5] Sebastien Bigo, Giovanni Bellotti and Michel W. Chbat, "Investigation of cross-phase modulation limitations on $10 \mathrm{Gbit} / \mathrm{s}$ transmission over various types of fiber infrastructures", IEEE Phot. Tech. Lett. 11, 1999, pp. 605-606

[6] H.J. Thiele, R. I. Killey and P. Bayvel, "Investigation of XPM Distortion in Transmission over Instralled Fiber", IEEE Phot. Tech. Lett. 12, 2000, 669-671

[7] Livio Paradiso, Pierpaolo Boffi, Lucia Marazzi, Giandomenico Pozzi, Massimo Artiglia and Mario Martinelli, "Electrical measure of Cross-Phase Modulation impact in WDM optical Systems", in Proceedings of Conference in Laser and Electro Optics, 2003, CFJ4 
[8] C. Fuerst, C. Scheerer, G. Mohs, J. P. Elbers, C. Glingener, "Influence of the dispersion map on limitations due to cross-phase modulation in WDM multispan transmission systems", Proceedings of Optical Fiber Conference, 2001, MF4-1

[9] C. Caspar, K. Habel, N. Heimes, M. Konitzer, M. Malach, H. Özdem, M.Rohde, F. Schmidt and E.-J. Bachus, "Penalties through XPM crosstalk in a switched long haul standard fiber WDM system based on normalized transmission sections", in Proceedings of Optical Fiber Conference, 2001, WI5-1 